\title{
Evaluating the Demand for Integrative Medicine Practices in Breast and Gynecological Cancer Patients
}

\author{
Nikolas Schuerger ${ }^{a} \quad E^{2}$ elyn Klein ${ }^{a} \quad$ Alexander Hapfelmeier ${ }^{b} \quad$ Marion Kiechle $^{a}$ \\ Christine Brambs ${ }^{a}$ Daniela Paepke ${ }^{a}$ \\ a Department of Gynecology and Obstetrics, Klinikum rechts der Isar, TU Munich, Munich, Germany; \\ ${ }^{\mathrm{b}}$ Institute of Medical Informatics, Statistics and Epidemiology, TU Munich, Munich, Germany
}

\section{Keywords}

Complementary and alternative medicine (CAM) . Integrative medicine · Breast cancer · Gynecological cancer

\begin{abstract}
Introduction: Integrative medicine is becoming increasingly relevant to modern oncology. The present study aims to assess the demand for integrative therapeutic approaches including complementary and alternative medicine (CAM), body and mind practices, nutritional counseling, and psychological assistance among breast and gynecological cancer patients. Methods: From April to July 2017, a pseudo-anonymous questionnaire was distributed to cancer patients at the Department of Gynecology and Obstetrics, Technical University of Munich. Patterns of CAM use and demand for integrative health approaches were investigated by generating descriptive statistics. Results: 182 (90\%) of 203 patients participated in the survey. $59 \%$ indicated the use of CAM practices in general, and $41 \%$ specifically in relation to their oncological disease. Most frequently used modalities were herbal supplements (32\%), homeopathy $(29 \%)$, vitamins $(28 \%)$, and selenium (21\%). Integrative health approaches that patients would have desired alongside conventional oncological treatment were CAM (54\%), relaxation therapies (44\%), and dietary counseling (43\%). Conclusion: There is a considerable demand for integrative medicine among breast and gynecological cancer patients. Our results underline the need for the implementation of evidence-based integrative treatments in cancer care in order to meet the standards of modern oncology.

(๑) 2018 S. Karger GmbH, Freiburg
\end{abstract}

\section{Introduction}

In the course of their disease, cancer patients often undergo immense physical and psychological distress due to pain, depression, anxiety, cachexia, or fatigue [1-4]. As conventional medical care does not always satisfactorily relieve these symptoms, patients frequently turn to complementary alternative medicine (CAM) concepts in their search for alleviation [5-7]. Research highlights various other reasons why cancer patients opt for health approaches which fall outside the sphere of conventional medical care: the wish to enhance the body's ability to fight the cancer, the desire to promote healing processes and to improve physical and emotional well-being, and the hope to augment the effectiveness of regular cancer therapy [5-8]. In response to that, the field of integrative oncology (IO) has emerged, a discipline that embraces conventional and evidence-based complementary therapies. IO represents a more holistic approach to oncological treatment [8-10] which considers all factors that affect health, including mind, emotions, lifestyle, and body $[9,11]$. The spectrum of IO is broad and may encompass supportive care services such as nutritional and psychological counseling or sports rehabilitation. However, many of the integrative health care interventions adopted by IO are part of the CAM family, a heterogeneous group of complementary healing modalities which the National Center for Complementary and Integrative Health (NCCIH) classifies into natural products (e.g., herbs, vitamins, minerals), mind and body-based practices (e.g., massage, yoga, meditation, acupuncture, osteopathic manipulation), and others (e.g., Ayurvedic medicine, traditional Chinese medicine, homeopathy) [12].

Cancer patients are increasingly using CAM, and the number of IO programs is growing internationally $[10,13,14]$. On average, about $40 \%$ of all people suffering from cancer in Western industri-

\section{KARGER}

(c) 2018 S. Karger GmbH, Freiburg

E-Mail karger@karger.com

www.karger.com/brc
Nikolas Schürger

St.-Veit-Straße 6, 412, 81673 München, Germany

nikolas.schuerger@gmx.de 
alized countries use CAM alongside their oncological treatment. It is noteworthy that women affected by gynecological malignancies appear to be particularly assiduous users $[6,7]$.

On the one hand, there is emerging evidence for the effectiveness of certain integrative health approaches in reducing treatment-related side effects, increasing quality of life, and mitigating cancer symptoms [15-18]. For instance, meta-analyses have proven physical exercise to be an effective intervention for cancerrelated fatigue, often also improving quality of life [19, 20]. Cancerrelated fatigue can also be successfully addressed by acupuncture $[21,22]$. Moreover, previous research has demonstrated positive effects of yoga on mental health, depression, sleep disturbances, and fatigue [23-25]. Up to $50 \%$ of cancer patients suffer from unintended and progressive weight loss with subsequent malnutrition [26]. A growing body of literature has established a close correlation between poor nutritional status and increased morbidity, hospitalization time, and costs. Malnourished patients appear to be more prone to nosocomial infections [27], have weaker responses to cancer therapy $[28,29]$ and lower quality of life $[30,31]$, and are associated with a trend for severe chemotherapy-induced toxicity [29] as well as increased treatment costs due to additional days of hospitalization [32]. These findings clearly underline the vital role dietary counseling should have in oncology, especially given the fact that a large number of malnourished patients receive no nutritional guidance [33].

On the other hand, not all integrative therapies are appropriate or beneficial. Especially in the case of CAM, simultaneous use alongside conventional therapy might interfere with or even compromise the effects of standard treatment, particularly since patients often do not disclose their CAM use to their oncologists [8, 34].

The present paper aims to investigate the demand for integrative therapeutic approaches among women suffering from gynecological malignancies in order to enable oncologists to better understand and address their specific needs. The prevalence of CAM use alongside conventional oncological treatment and the most commonly applied modalities were evaluated in order to gain additional information on patterns of CAM usage among cancer patients.

\section{Material and Methods}

The survey was conducted from April to July 2017 by means of a structured questionnaire at the Department of Gynecology and Obstetrics (OBGYN), Technical University Munich (TUM), Munich, Germany, distributed to inpatients diagnosed with breast and gynecological cancer.

Inclusion criteria were age $\geq 18$ years, command of the German or English language, and the mental ability to fill out a questionnaire.

The German survey questionnaire comprised 38 items and was developed by the authors based on previous research in the field of gynecological oncology. We pretested the set of closed-ended questions using a convenience sample of 10 patients. The final questionnaire consisted of 3 sections: i) personal use and opinions regarding CAM; ii) attitudes, interest, and demand for integrative therapeutic approaches alongside conventional medical care; and iii) assessment of sociodemographic factors and health behaviors.
Table 1. Tumor entities among the study population

\begin{tabular}{lc}
\hline Diagnosis & Patients, n (\%) \\
\hline Breast cancer & $129(70.8)$ \\
Ovarian cancer & $32(17.6)$ \\
Cervical cancer & $11(6.0)$ \\
Endometrial cancer & $6(3.3)$ \\
Vulvar cancer & $4(2.2)$ \\
\hline
\end{tabular}

Participation was voluntary and all data were collected in pseudo-anonymous form. Informed consent was given. Participants filled out the questionnaires autonomously; however, if they were English-speaking only or preferred assistance, it was completed within a face-to-face interview. The final version was approved by the TUM Ethics Committee. No incentive was offered for study participation.

\section{Statistical Analysis}

Descriptive statistics were generated to determine the prevalence and patterns of CAM use as well as the demand for integrative therapy approaches. Univariate $\mathrm{t}$ - and chi-squared tests were conducted for hypothesis testing on associations between interest in integrative therapy options and the application of CAM on the one hand, and sociodemographic characteristics and health behaviors on the other. An exploratory data analysis was performed without a specific a priori hypothesis. Patients with missing values were excluded from the analysis of corresponding variables. Hypothesis testing was conducted on a 2 -sided significance level of 5\%. Statistical analyses were performed using SPSS software, version 20 (IBM Corp., Armonk, NY, USA).

\section{Results}

A total of 182 out of 203 patients participated in the survey (return rate of $90 \%$ ). Mean age was 58.2 years (standard deviation 14.4 years). $59 \%(\mathrm{n}=107)$ of the respondents reported using CAM in general, $41 \%(\mathrm{n}=74)$ specifically for their oncological disease, and $56 \%(\mathrm{n}=102)$ in the past. Most patients $(71 \%)$ included in our study suffered from breast cancer (table 1).

Figure 1 depicts the CAM modalities and relaxation therapies most frequently used by participants. Herbs were the most used category (32\%), followed by homeopathy (29\%), and vitamins (28\%).

The majority of respondents (38\%) indicated family and friends as the most relevant source of information (fig. 2).

A total of $45 \%$ reported sourcing and performing CAM products and treatments by themselves, 38\% through non-medical practitioners, 27\% through physicians at our OBGYN department, $18 \%$ through their general practitioner, and $8 \%$ through their oncologist.

A total of $60 \%$ of CAM users reported an overall good effect of the CAM practices or products, $14 \%$ classified it as moderate, $22 \%$ as uncertain, and $4 \%$ as non-existent.

Figure 3 shows the demand for integrative therapeutic approaches alongside conventional oncological treatment. Slightly more than half of the participants $(54 \%, \mathrm{n}=98)$ expressed the wish for implementation of CAM into cancer care and declared that they would have taken advantage of it had it been part of the regular cancer treatment services. 


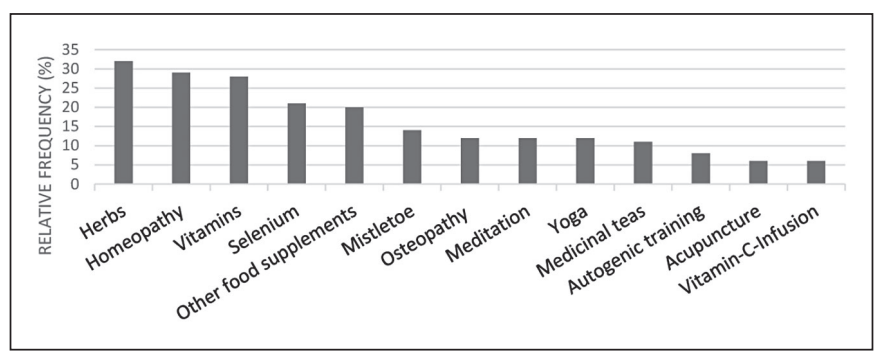

Fig. 1. Most frequently used complementary and alternative medicine (CAM) modalities and relaxation therapies alongside oncological treatment. Relative frequencies reported in relation to the total sample size $(n=182)$. Multiple responses were possible.

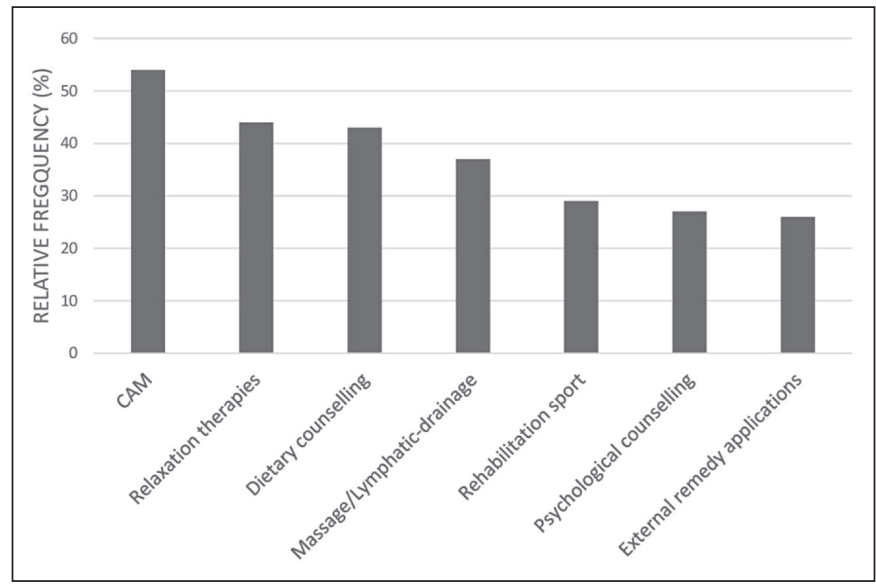

Fig. 3. Demand for integrative therapeutic approaches among the study population $(\mathrm{n}=182)$. Multiple responses were possible.

In order to determine which factors were associated with the use of CAM and the demand for integrative therapies, univariate analyses were performed (table 2). On average, CAM use was more frequent in patients who were younger, employed, regularly engaging in physical activity, or who had higher levels of education. An increased demand for CAM, relaxation therapies, and nutritional counseling was also observed in participants who were currently using CAM and were on average younger. Women with a potential interest in CAM and relaxation therapies had higher levels of education. Interest in CAM and dietary counseling was shown to be decreased among unemployed women. Lastly, patients who were younger, highly educated, and employed were more likely to demand psychological counseling.

\section{Discussion}

In this study, we surveyed breast and gynecological cancer patients on their usage of CAM and explored their perspectives on and demand for integrative health approaches. The majority of participants were using CAM in addition to their conventional on-

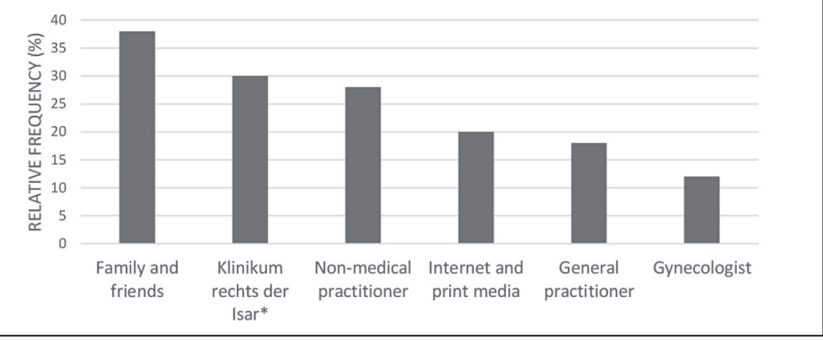

Fig. 2. Sources of recommendation with the strongest influence on participants regarding complementary and alternative medicine (CAM) use alongside oncological treatment. Multiple responses were possible. *Department of OBGYN.

cological treatment, and dietary supplements were the most prevalent category. Sources of recommendation and provision of CAM modalities were predominantly non-professional. Moreover, our results confirm a high unmet demand for integrative interventions among women suffering from gynecological malignancies.

The scarce coverage of the demand for supportive care services is a phenomenon not seldom observed in the literature $[5,6,35$, 36]. Despite conspicuous interest among patients as well as growing acceptance among physicians, a persistent lack of implementation of integrative medicine into standard cancer care in Germany has been reported [37]. Hence, it is not astonishing that patients often turn to non-professional sources for complementary treatments $[7,34,38,39]$. Our results confirm this inasmuch as family/ friends and non-medical practitioners were 2 of the 3 main sources of information and recommendation regarding CAM. Gaining access to CAM mainly through non-professional sources and providers is problematic since uncritical usage might involve a potential for interactions between conventional and complementary therapies, particularly in the case of herbal supplements [40-42]. Overall, $59 \%$ of our participants reported usage of CAM, mostly dietary supplements, alongside their oncological therapy, which is consistent with previous studies $[6,35,36,38,39]$. Nevertheless, prevalence rates vary widely throughout the literature, mostly due to the lack of a consistent definition of CAM and associated modalities, as well as differences in ethnic and cultural attitude towards complementary health approaches. Following the example of previous studies, we referred to CAM according to its $\mathrm{NCCIH}$ definition [6, $43,44]$, and we invite future researchers to do the same in order to establish a uniform definition of CAM.

To our knowledge, this is one of the first studies to assess the demand for different integrative supportive services among women with breast and gynecological cancer.

Slightly more than half (54\%) of the surveyed women expressed their support for the implementation of CAM into conventional cancer care. Patients' characteristics significantly associated with the wish for integration of complementary healing approaches are mainly the same, namely age, education, employment, and usage of CAM. This is concordant with the literature $[5,6,8,39]$ and suggests that patients inclined towards one of the supportive services are very likely to be interested in integrative medicine in general. 


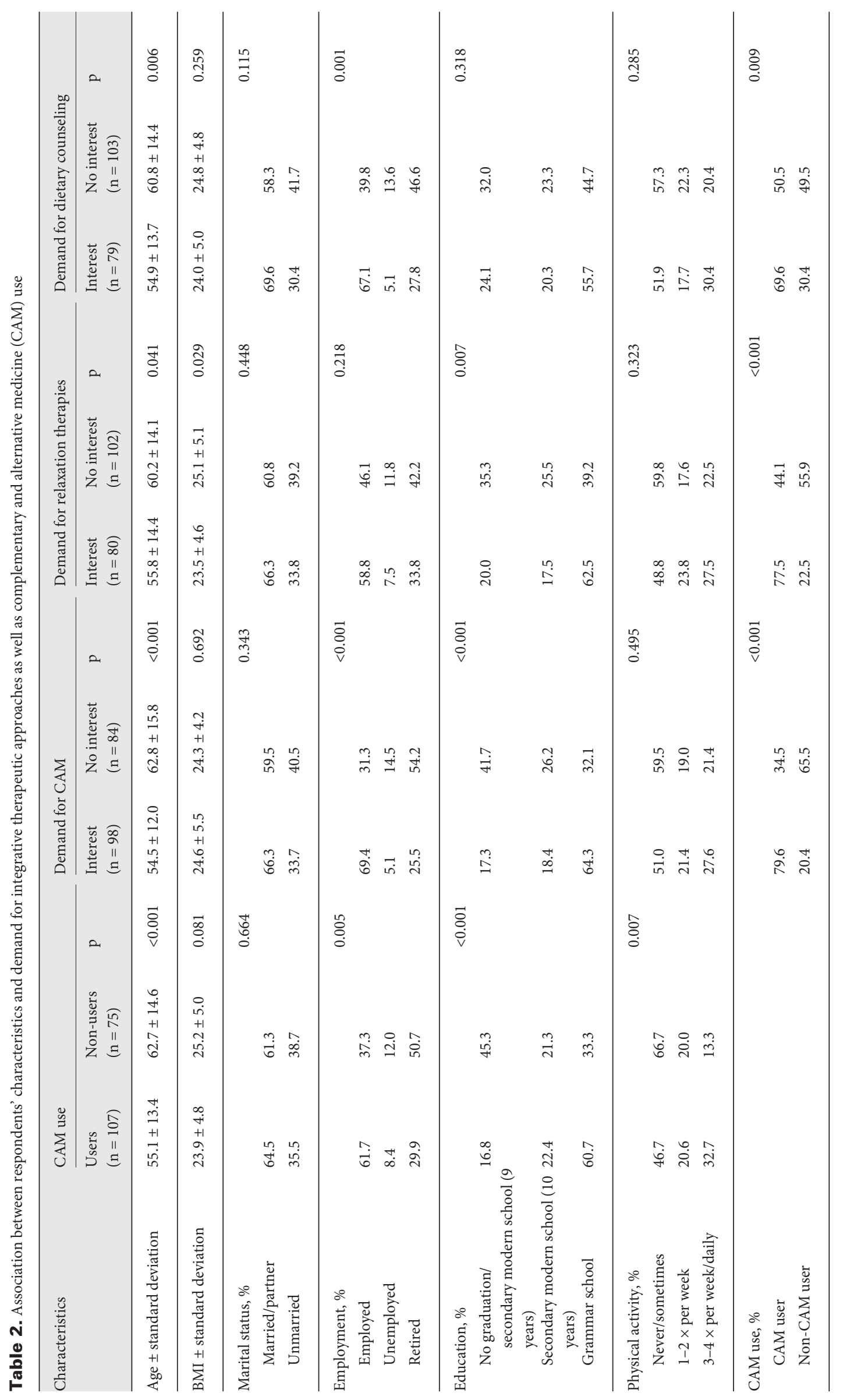


Nutritional counseling was offered to only $12 \%(n=22)$ of our study population, whereas $43 \%(n=79)$ would have been interested in it. Despite the progressive improvement in cancer care over the past decades, and although the beneficial effects of dietary assistance are extensively documented in the literature [45-47], malnutrition is still prevalent among cancer patients [26, 33, 48]. Our results in line with previous studies confirm a low rate of supply [33, 48], clearly highlighting the importance nutritional counseling should have in oncological treatment.

Nearly half (44\%) of the surveyed women expressed the wish for relaxation therapies as part of the regular therapy services; a further 37 and $27 \%$ did so with regard to massage/lymphatic drainage and psychological counseling, respectively. Since cancer affects not only the body but also the psyche and mind, modern oncology needs to pay due attention to psychological and mental aspects. Fortunately, psychological counseling is incontestably of great importance in state-of-the-art cancer care $[49,50]$. Approximately half (46\%) of our participants had been offered psycho-oncological assistance, while $23 \%$ of those who had not been interrogated on their need for it declared that they would have taken advantage of it had they been asked. The difficulty of screening cancer patients regarding psychosocial intervention is well known $[51,52]$. Since the effectiveness of massages, yoga, and psychological assistance in promoting mental health has been assessed [23-25, 53, 54], modern oncology should implement such interventions in order to ensure the highest possible psychological well-being among cancer patients.

Over the past 2 decades, the number of certified breast cancer centers has been steadily growing in Germany. With the aim in mind to provide multidisciplinary, comprehensive, and qualitycontrolled care, the German Cancer Society and the German Society of Senology initiated a voluntary breast center certification process in 2003 [55]. It is noteworthy that from the integrative supportive services we evaluated in this survey, psychological care is the only mandatory integrative supportive service a certified breast center has to provide. Interestingly enough, only $27 \%$ of our study population expressed the wish for implementation of psychological counseling into cancer care, whereas $54 \%$ did so with respect to CAM concepts and $43 \%$ to dietary counseling. Hack et al. [56] were aware of breast cancer patients' growing interest in integrative therapeutic approaches but came to realize that published data on how to implement a comprehensive consultancy service on integrative medicine were missing. Hence, they developed a standardized procedure for conducting an integrative medicine consultation and incorporating it into routine patient care at the Department of OBGYN in Erlangen, Germany. Another successful example of an IO model in Germany is the breast care center at the 'Kliniken Essen-Mitte', Duisburg [57], where individualized treatment plans are offered to patients, combining standard cancer care with integrative supportive care services. As practical concepts of IO start to emerge in Germany, it might be worth considering extending the spectrum of integrative therapeutic approaches in certified breast centers; ideally, patients should be offered counseling on integrative medicine, and treating oncologists should receive basic training in it. Meanwhile, the website of the Gynecological Oncology Working Group (Arbeitsgemeinschaft Gynäkologische Onkologie) not only offers information on several complementary health approaches in the treatment of breast cancer patient, but it is also working on a curriculum for evidence-based training in integrative medicine for oncologists [58].

When interpreting our study results, certain limitations should be taken into account. Firstly, due to its self-reporting nature, our survey data is prone to recall bias. Secondly, the survey was conducted monocentrically and therefore may not be representative of other parts of Germany. Thirdly, we included all patients diagnosed with breast and gynecological cancer regardless of their disease stage. Different stages and phases of a disease may result in different profiles of demand for integrative supportive services.

\section{Conclusion}

Our results demonstrate a considerable demand for integrative medicine among breast and gynecological cancer patients which continues to be insufficiently addressed by conventional medicine. Once again, this affirms our efforts to implement evidence-based treatments into clinical routine in addition to established cancer therapies in order to meet the standards of modern oncology and to comprehensively address patients' needs.

\section{Author Contribution Statement}

NS: Conceptualization, data curation, formal analysis, project administration, writing (original draft, review/editing), visualization; EK: conceptualization, supervision, writing (review/editing); $\mathrm{AH}$ : data curation, formal analysis, methodology, visualization, writing (review/editing); DP: conceptualization, supervision, project administration, writing (review/editing); MK: conceptualization, methodology, supervision, writing (review/editing); CP: writing (review/editing).

\section{Ethical Statement}

The final version was approved by the Ethics Committee of the Technical University of Munich (project no. 73/17 S). A preliminary statement on the front of the questionnaire informed patients about the voluntary and pseudoanonymous nature of the study as well as its purpose.

\section{Disclosure Statement}

The authors declare that they have no competing interests. The authors received no specific funding for this work. 


\section{References}

1 Krebber AM, Buffart LM, Kleijn G, et al: Prevalence of depression in cancer patients: a meta-analysis of diagnostic interviews and self-report instruments. Psychooncology 2014;23:121-130.

2 Mortimer JE, Barsevick AM, Bennett CL, et al: Studying cancer-related fatigue: report of the NCCN scientific research committee. J Natl Compr Canc Netw 2010;8:1331-1339.

3 Penet MF, Bhujwalla ZM: Cancer cachexia, recent advances, and future directions. Cancer J 2015;21:117122.

4 Van den Beuken-van Everdingen MH, de Rijke JM, Kessels AG, et al: Prevalence of pain in patients with cancer: a systematic review of the past 40 years. Ann Oncol 2007;18:1437-1449.

5 Hack CC, Fasching PA, Fehm T, et al: Interest in integrative medicine among postmenopausal hormone receptor-positive breast cancer patients in the EvAluateTM study. Integr Cancer Ther 2017;16:165-175.

6 Lettner S, Kessel KA, Combs SE: Complementary and alternative medicine in radiation oncology: survey of patients' attitudes. Strahlenther Onkol 2017;193:419-425.

7 Molassiotis A, Fernadez-Ortega P, Pud D, et al: Use of complementary and alternative medicine in cancer patients: a European survey. Ann Oncol 2005;16:655-663.

$\checkmark$ Kalder M, Muller T, Fischer D, et al: A review of integrative medicine in gynaecological oncology. Geburtshilfe Frauenheilkd 2016;76:150-155.

9 Leis AM, Weeks LC, Verhoef MJ: Principles to guide integrative oncology and the development of an evidence base. Curr Oncol 2008; 15:s83-s87.

10 Seely D, Weeks L, Young S: A systematic review of integrative oncology programs. Curr Oncol 2012; 19:e436-461.

11 Maizes V, Rakel D, Niemiec C: Integrative medicine and patient-centered care. Explore (NY) 2009;5:277-289.

12 National Center for Complementary and Integrative Health (NCCIH): Complementary, Alternative, or Integrative Health: What's In a Name? 2015. nccih.nih. gov/health/integrative-health.

13 Cramer H, Cohen L, Dobos G, Witt CM: Integrative Oncology: Best of both worlds - theoretical, practical, and research issues. Evid Based Complement Alternat Med 2013;2013:383142.

14 Horneber M, Bueschel G, Dennert G, et al: How many cancer patients use complementary and alternative medicine: a systematic review and metaanalysis. Integr Cancer Ther 2012;11:187-203.

15 Blaes AH, Kreitzer MJ, Torkelson C, Haddad T: Nonpharmacologic complementary therapies in symptom management for breast cancer survivors. Semin Oncol 2011;38:394-402.

16 Deng G, Cassileth BR: Integrative oncology: complementary therapies for pain, anxiety, and mood disturbance. CA Cancer J Clin 2005;55:109-116.

17 Fasching PA, Thiel F, Nicolaisen-Murmann K, et al: Association of complementary methods with quality of life and life satisfaction in patients with gynecologic and breast malignancies. Support Care Cancer 2007; 15:1277-1284

18 Finnegan-John J, Molassiotis A, Richardson A, Ream E: A systematic review of complementary and alternative medicine interventions for the management of cancer-related fatigue. Integr Cancer Ther 2013;12: 276-290.

19 Cramp F, Byron-Daniel J: Exercise for the management of cancer-related fatigue in adults. Cochrane $\mathrm{Da}-$ tabase Syst Rev 2012;11:CD006145.

20 McNeely ML, Campbell KL, Rowe BH, et al: Effects of exercise on breast cancer patients and survivors: a systematic review and meta-analysis. CMAJ 2006;175: $34-41$.
He XR, Wang Q, Li PP: Acupuncture and moxibustion for cancer-related fatigue: a systematic review and metaanalysis. Asian Pac J Cancer Prev 2013;14:3067-3074.

22 Ling WM, Lui LY, So WK, Chan K: Effects of acupuncture and acupressure on cancer-related fatigue: a systematic review. Oncol Nurs Forum 2014;41:581-592.

23 Danhauer SC, Mihalko SL, Russell GB, et al: Restorative yoga for women with breast cancer: findings from a randomized pilot study. Psychooncology 2009;18:360-368.

24 Mustian KM, Palesh O, Sprod L, et al: Effect of YOCAS yoga on sleep, fatigue, and quality of life: A URCC CCOP randomized, controlled clinical trial among 410 cancer survivors. J Clin Oncol 2010;28:9013.

25 Sadja J, Mills PJ: Effects of yoga interventions on fatigue in cancer patients and survivors: a systematic review of randomized controlled trials. Explore (NY) 2013;9:232-243.

26 August DA, Huhmann MB: A.S.P.E.N. clinical guidelines: nutrition support therapy during adult anticancer treatment and in hematopoietic cell transplantation. JPEN J Parenter Enteral Nutr 2009;33:472-500.

27 Schneider SM, Veyres P, Pivot X, et al: Malnutrition is an independent factor associated with nosocomial infections. Br J Nutr 2004;92:105-111.

28 Murry DJ, Riva L, Poplack DG: Impact of nutrition on pharmacokinetics of anti-neoplastic agents. Int J Cancer Suppl 1998;11:48-51.

29 Van Cutsem E, Arends J: The causes and consequences of cancer-associated malnutrition. Eur J Oncol Nurs 2005;9(suppl 2):S51-63.

30 Lis CG, Gupta D, Lammersfeld CA, Markman M, Vashi PG: Role of nutritional status in predicting quality of life outcomes in cancer - a systematic review of the epidemiological literature. Nutr J 2012;11:27.

31 Marin Caro MM, Laviano A, Pichard C: Impact of nutrition on quality of life during cancer. Curr Opin Clin Nutr Metab Care 2007;10:480-487.

32 Tucker HN, Miguel SG: Cost containment through nutrition intervention. Nutr Rev 1996;54:111-121.

33 Hebuterne X, Lemarie E, Michallet M, et al: Prevalence of malnutrition and current use of nutrition support in patients with cancer. JPEN J Parenter Enteral Nutr 2014;38:196-204.

34 Koehl B, Muenstedt K, Micke O, et al: Survey of German non-medical practitioners regarding complementary and alternative medicine in oncology. Oncol Res Treat 2014;37:49-53.

35 Ben-Arye E, Schiff E, Steiner M, Keshet Y, Lavie O: Attitudes of patients with gynecological and breast cancer toward integration of complementary medicine in cancer care. Int J Gynecol Cancer 2012;22:146-153.

36 Fremd C, Hack CC, Schneeweiss A, et al: Use of complementary and integrative medicine among German breast cancer patients: predictors and implications for patient care within the PRAEGNANT study network. Arch Gynecol Obstet 2017;295:1239-1245.

37 Klein E, Beckmann MW, Bader W, et al: Gynecologic oncologists' attitudes and practices relating to integrative medicine: results of a nationwide AGO survey. Arch Gynecol Obstet 2017;296:295-301.

38 Loquai C, Dechent D, Garzarolli M, et al: Use of complementary and alternative medicine: a multicenter cross-sectional study in 1089 melanoma patients. Eur J Cancer 2017;71:70-79.

39 Wanchai A, Armer JM, Stewart BR: Complementary and alternative medicine use among women with breast cancer: a systematic review. Clin J Oncol Nurs 2010;14:E45-55.

40 Beijnen JH, Schellens JH: Drug interactions in oncology. Lancet Oncol 2004;5:489-496.

41 Meijerman I, Beijnen JH, Schellens JH: Herb-drug interactions in oncology: focus on mechanisms of induction. Oncologist 2006;11:742-752.
Zeller T, Muenstedt K, Stoll C, et al: Potential interactions of complementary and alternative medicine with cancer therapy in outpatients with gynecological cancer in a comprehensive cancer center. J Cancer Res Clin Oncol 2013;139:357-365.

43 Birdee GS, Kemper KJ, Rothman R, Gardiner P: Use of complementary and alternative medicine during pregnancy and the postpartum period: an analysis of the National Health Interview Survey. J Womens Health (Larchmt) 2014;23:824-829.

44 Kalder M, Knoblauch K, Hrgovic I, Munstedt K: Use of complementary and alternative medicine during pregnancy and delivery. Arch Gynecol Obstet 2011;283:475-482.

45 Isenring EA, Capra S, Bauer JD: Nutrition intervention is beneficial in oncology outpatients receiving radiotherapy to the gastrointestinal or head and neck area. Br J Cancer 2004;91:447-452.

46 Langius JA, Zandbergen MC, Eerenstein SE, et al: Effect of nutritional interventions on nutritional status, quality of life and mortality in patients with head and neck cancer receiving (chemo)radiotherapy: a systematic review. Clin Nutr 2013;32:671-678.

47 Ravasco P, Monteiro-Grillo I, Vidal PM, Camilo ME: Dietary counseling improves patient outcomes: a prospective, randomized, controlled trial in colorectal cancer patients undergoing radiotherapy. J Clin Oncol 2005;23:1431-1438.

48 Pressoir M, Desne S, Berchery D, et al: Prevalence, risk factors and clinical implications of malnutrition in French Comprehensive Cancer Centres. Br J Cancer 2010;102:966-971.

49 Institute of Medicine Committee on Psychosocial Services to Cancer Patients/Families in a Community Setting - The National Academies Collection: Reports funded by National Institutes of Health; Adler NE, Page AEK (eds): Cancer Care for the Whole Patient: Meeting Psychosocial Health Needs. Washington (DC), National Academies Press (US), 2008.

50 Lazenby M: The international endorsement of US distress screening and psychosocial guidelines in oncology: a model for dissemination. J Natl Compr Cancer Netw 2014;12:221-227.

51 Baker-Glenn EA, Park B, Granger L, Symonds P, Mitchell AJ: Desire for psychological support in cancer patients with depression or distress: validation of a simple help question. Psychooncology 2011;20:525-531.

52 Merckaert I, Libert Y, Messin S, et al: Cancer patients desire for psychological support: prevalence and implications for screening patients' psychological needs. Psychooncology 2010;19:141-149.

53 Jassim GA, Whitford DL, Hickey A, Carter B: Psychological interventions for women with non-metastatic breast cancer. Cochrane Database Syst Rev 2015;CD008729.

54 Ovayolu O, Sevig U, Ovayolu N, Sevinc A: The effect of aromatherapy and massage administered in different ways to women with breast cancer on their symptoms and quality of life. Int J Nurs Pract 2014;20:408-417.

55 Senologie DGf: Zertifizierungsrichtlinien. www.senologie.org/brustzentren/zertififzierungsrichtlinien.

56 Hack CC, Antoniadis S, Hackl J, et al: Breast cancer patients' satisfaction with individual therapy goals and treatment in a standardized integrative medicine consultancy service. Arch Gynecol Obstet 2018;298:147-156.

57 Kliniken Essen-Mitte B: Integrative Onkologie: Naturheilkunde und Mind-Body Medizin in der onkologischen Behandlung. kliniken-essen-mitte.de/leistung/ fachabteilungen/senologie-brustzentrum/leistungenund-angebote/naturheilkunde.html.

58 AGO e.V.: Empfehlungen gynäkologische Onkologie Kommission Mamma, 2016. www.ago-online.de/de/ infothek-fuer-aerzte/leitlinienempfehlungen/mamma/. 